\title{
Efficacy of a Self-Expanding Tract Sealant Device in the Reduction of Pneumothorax and Chest Tube Placement Rates after Percutaneous Lung Biopsy: A Matched Controlled Study Using Propensity Score Analysis
}

\author{
Judy U. Ahrar, M.D., Sanjay Gupta, M.D., Joe E. Ensor, Ph.D., Armeen Mahvash, M.D., \\ Sharjeel H. Sabir, M.D., Joseph R. Steele, M.D., Stephen E. McRae, M.D., Rony Avritscher, \\ M.D., Steven Y. Huang, M.D., Bruno C. Odisio, M.D., Ravi Murthy, M.D., Kamran Ahrar, M.D., \\ Michael J. Wallace, M.D., and Alda L. Tam, M.D. \\ University of Texas MD Anderson Cancer Center, Department of Interventional Radiology, \\ Houston, TX (J.U.A., A.M., S.G., A.M., S.H.S., J.R.S., S.E.M., R.A., S.Y.H., B.C.O., R.M., K.A., \\ M.J.W., A.L.T.); and the Houston Methodist Research Institute, Houston Methodist Cancer Center, \\ Houston, TX (J.E.E.)
}

\section{Abstract \\ Purpose-To evaluate the use of a self-expanding tract sealant device (BioSentry $\left.{ }^{\mathrm{TM}}\right)$ on the rates of pneumothorax and chest tube insertion after percutaneous lung biopsy.}

Material and Methods-In this retrospective study, we compared 318 patients who received BioSentry ${ }^{\mathrm{TM}}$ during percutaneous lung biopsy (treated group) with 1956 patients who did not (control group). Patient-, lesion-, and procedure-specific variables, and pneumothorax and chest tube insertion rates were recorded. To adjust for potential selection bias, patients in the treated group were matched 1:1 to patients in the control group using propensity score matching based on the above-mentioned variables. Patients were considered a match if the absolute difference in their propensity scores was sequal to 0.02 .

Results-Before matching, the pneumothorax and chest tube rates were $24.5 \%$ and $13.1 \%$ in the control group, and $21.1 \%$ and $8.5 \%$ in the treated group, respectively. Using propensity scores, a match was found for 317 patients in the treatment group. Chi-square contingency matched pair analysis showed the treated group had significantly lower pneumothorax (20.8\% vs. 32.8\%; $\mathrm{p}=$. $001)$ and chest tube ( $8.2 \%$ vs. $20.8 \%$; $<$ <.0001) rates compared to the control group. Sub-analysis including only faculty who had $>30$ cases of both treatment and control cases demonstrated similar findings: the treated group had significantly lower pneumothorax $(17.6 \%$ vs. $30.2 \% ; \mathrm{p}=$. $002)$ and chest tube $(7.2 \%$ vs. $18 \% ; \mathrm{p}=.001)$ rates.

Correspondence to: Judy Ahrar, M.D., Department of Interventional Radiology, Unit 1471, The University of Texas M.D. Anderson Cancer Center, PO Box 301402, Houston, Texas 77230-1402, judy.ahrar@mdanderson.org.

Oral abstract presentation SIR 2015

CONFLICT OF INTEREST

Authors \#1-13 declare they have no conflict of interest. Author \#14 is a medical monitor for Galil Medical and receives research support from Angiodynamics. 
Conclusions-The self-expanding tract sealant device significantly reduced the pneumothorax rate, and more importantly, the chest tube placement rate after percutaneous lung biopsy.

\section{INTRODUCTION}

Pneumothorax is a common complication following percutaneous computed tomographic (CT)-guided lung biopsy and can lead to additional radiographs, prolonged observation time, and occasionally hospital admission and chest tube placement.[1,2] Large CT-guided lung biopsy series report rates of pneumothorax ranging from $15-25 \%,[3-7]$ and rates of postbiopsy chest tube placement ranging from 0-17\%.[4-7] Various methods for sealing the biopsy tract to mitigate pneumothorax occurrence have been investigated without the emergence of a singular technique that has been widely accepted or adopted [8]. The types of sealants that have been studied in both the pre-clinical and clinical trial settings include saline, autologous blood patch, collagen or hydrogel plugs, and fibrin glue.[9-19]

The hydrogel plug, US Food and Drug Administration (FDA) approved and currently marketed as BioSentry ${ }^{\mathrm{TM}}$ (Surgical Specialties Corporation, Wyomissing, PA), has been shown in a prospective clinical trial to reduce the rates of pneumothorax occurrence and chest tube placement following CT-guide lung biopsy.[19] However, this study was performed 7 years ago, with the product only recently receiving FDA approval and becoming available for clinical use. We wanted to confirm the results of the trial in clinical practice among multiple faculty with variable experience. The objective of our study was to evaluate the effect of using the self-expanding tract sealant device (BioSentry ${ }^{\mathrm{TM}}$ ) on the rates of pneumothorax and chest tube placement after percutaneous lung biopsy in oncology patients. We hypothesized that use of the BioSentry ${ }^{\mathrm{TM}}$ device after lung biopsy would lower pneumothorax and chest tube insertion rates.

\section{MATERIALS AND METHODS}

The Institutional Review Board approved this HIPAA compliant, retrospective review and granted a waiver of consent. A total of 2,274 CT-guided lung biopsies were performed between 8/20/2007 and 7/31/14 at our institution. Biopsies that required the needle trajectory to cross a pleural surface were included in the study and those that did not require transgression of a pleural surface were excluded. BioSentry ${ }^{\mathrm{TM}}$ device became available for clinical use at our institution starting June, 2013; the decision of when to place the device was based on operator preference.

A co-axial technique, with a 19-gauge guide needle, was used for all CT-guided lung biopsies. One of twelve board-certified interventional radiologists (experience range, 2-15 years), either acting as an independent operator or directly supervising a resident or fellow, performed all procedures. Conscious sedation or sedation provided by the Anesthesia service was administered to all patients. Tissue sampling consisted of fine needle aspiration (FNA) with a 22-gauge needle and/or acquisition of a core biopsy specimen using a 20-gauge automated biopsy needle.

Our outpatient post-biopsy pneumothorax management protocol has been previously described.[1, 20, 21] In brief, after the CT-guided lung biopsy, patients were observed in a 
monitored recovery unit for a minimum of three hours and the presence of pneumothorax was assessed using serial chest radiographs. Based on our management algorithm, small, stable and asymptomatic pneumothoraces are treated conservatively without chest tube insertion. Pneumothoraces that rapidly expand, cause symptoms of chest pain or shortness of breath, or are large ( $\geq 30 \%$ of the hemithorax) are treated with the placement of a small bore chest tube (8.5 French, Richili tube, Cook Inc., Bloomington, IN).

\section{Statistical Methods}

The rates of pneumothorax occurrence and chest tube insertion were compared between two groups of patients who underwent CT-guided lung biopsy: patients receiving the BioSentry ${ }^{\mathrm{TM}}$ device $(\mathrm{n}=318)$ [treatment group] versus patients not receiving the BioSentry ${ }^{\mathrm{TM}}$ device ( $n=1956)$ [control group]. Ten variables were selected for analysis. Patient-specific variables were age, sex, and the presence or absence of emphysema in the biopsy needle path. Lesion-specific variables were lesion size and location. Procedure-specific variables were type of biopsy obtained (FNA versus core biopsy versus both), needle path length, number of pleural surfaces crossed to access the lesion, patient position during the biopsy (prone, supine, decubitus), and biopsy approach to the lesion (anterior, posterior, or lateral). The needle path length was measured as the distance along the guide needle shaft from the point of pleural puncture to the guide needle tip and the number of pleural surfaces crossed was calculated by adding the number of times a visceral pleural surface was transgressed. [21]

A major disadvantage of retrospective or observational studies is selection bias or the possibility that systematic differences between baseline characteristics of the treated group versus control group of patients could account for the results of the study. Propensity score methods, statistical means of controlling for confounding non-experimental variables, permit the analysis of an observational study as if it were a randomized controlled trial. The purpose of propensity score methods is to achieve balance; that is, conditional on the propensity score, the distribution of baseline covariates should be homogeneous between the treated and untreated patients and account for systematic differences in baseline characteristics between the two patient groups when estimating the effect of the treatment.

To correct for potential selection bias in this study, the data were analyzed using propensity score matching. The propensity score model was created using a multivariate logistic regression model fitted to the data with the response being BioSentry ${ }^{\mathrm{TM}}$ device (Yes $=1$ vs. $\mathrm{No}=0$ ) (i.e., treated vs. untreated) and the model covariates being the ten data variables collected representing baseline characteristics. The propensity score for each patient was the estimated probability of being treated derived from the model. Propensity score matching was used to obtain matched (1:1) samples of treated and untreated patients. Patients were considered a match if the absolute difference in their propensity scores was $\leq 0.02$. Of the 318 patients who received a BioSentry ${ }^{\mathrm{TM}}$ device, a match was found for 317 patients. A subanalysis was performed which included only interventional radiology faculty who had $\geq 30$ CT-guided lung biopsy cases in which the BioSentry ${ }^{\mathrm{TM}}$ device was used and that could be matched to equal number of their own control cases. In this subanalysis, five faculty met the criteria, resulting in the identification of 222 matched cases. Chi-square contingency table 
evaluated with McNemar's test, which is appropriate for paired data, was used to analyze the matched samples for differences in pneumothorax and chest tube insertion rate. Statistical significance was defined as $\mathrm{p}<0.05$ and all analyses were conducted using SAS (Release 9.4, 2013, SAS Institute: Cary, NC).

\section{RESULTS}

The propensity score model was created by fitting a multivariate logistic regression model of 10 covariates to data (Table 1). While all ten variables were included in the model, only smaller lesions $(\mathrm{p}=.01)$, cases in which a core biopsy was obtained $(\mathrm{p}<.0001)$, presence of emphysema ( $\mathrm{p}<.0001)$, and lateral approach to biopsy $(\mathrm{p}=.007)$ were found to be significantly different between the two treatment groups. The model fits the data adequately, as the area under the curve (AUC) is .659.

Before matching, the pneumothorax and chest tube insertion rates were $24.5 \%$ and $13.1 \%$ in the control group, and $21.1 \%$ and $8.5 \%$ in the treated group, respectively. The unmatched and matched descriptive statistics of the observed baseline patient-, lesion-, and procedurespecific characteristics are reported (Table 2). The unmatched data (Table 2) demonstrate that the significant factors in the multivariate logistic regression analysis were not balanced between the two groups with the greatest degree of difference occurring in the variables of biopsy type and presence of emphysema. Using propensity scores, a match was found for 317 patients in the treatment group, bringing the descriptive statistics for the two groups closer together (absolute difference in their propensity scores was $\leq 0.02$ ). Chi-square contingency matched pair analysis showed: the treated group had significantly lower pneumothorax (20.8\% vs. $32.8 \%$; $\mathrm{p}=.001)$ and chest tube $(8.2 \%$ vs. $20.8 \%$; $\mathrm{p}<.0001)$ rates as compared to the control group (Table 3 ).

Results of a further sub analysis of the 222 cases performed by faculty who had at least 30 propensity score matched cases in both treatment groups are shown in table 4. Chi-square contingency matched pair analysis demonstrated that treated group had significantly lower pneumothorax (17.6\% vs. $30.2 \% ; \mathrm{p}=.002)$ and chest tube rates $(7.2 \%$ vs. $18 \% ; \mathrm{p}=.001)$ (Table 5). There were no reported adverse events related to the use of the BioSentry ${ }^{\mathrm{TM}}$ device.

\section{DISCUSSION}

In our study, which uses propensity scoring to match patients who received BioSentry ${ }^{\mathrm{TM}}$ to appropriate control patients, the use of the BioSentry ${ }^{\mathrm{TM}}$ tract sealant after lung biopsies was associated with significant decreases in pneumothorax and chest tube insertion rates. These results validate the prospective, multicenter, randomized, controlled clinical study published in 2010 by Zaetta et al. which demonstrated use of the BioSentry ${ }^{\mathrm{TM}}$ device (previously known as the Bio-Seal Lung Biopsy Tract Plug) significantly reduced rates of pneumothorax occurrence.[19] The study also demonstrated decreases in chest tube placement rates, and post-procedural hospital admissions however these secondary endpoints were not powered sufficiently to detect statistical significance.[19] Our results also appear to mirror those of Grage et al. who retrospectively compared the use of the BioSentry ${ }^{\mathrm{TM}}$ Tract Sealant Device 
in 100 consecutive patients who underwent percutaneous lung biopsy to a prior group of 100 lung biopsy patients who did not receive BioSentry ${ }^{\mathrm{TM}}$.[22] They concluded that although there was no difference in the pneumothorax rate between the control versus the BioSentry ${ }^{\mathrm{TM}}$ groups (31\% vs. $30 \%$, respectively), they did observe a reduction in chest tube insertion rates ( $10 \%$ for control vs. $2 \%$ for BioSentry $\left.{ }^{\mathrm{TM}}\right)$ and average length of stay in the hospital (0.44 days for control vs. 0.07 days for BioSentry $\left.{ }^{\mathrm{TM}}\right)$.[22]

The concept of sealing the lung biopsy tract as a strategy to prevent pneumothorax has been the subject of multiple research investigations.[9-19] The major difference between the studies that are now emerging that use the BioSentry ${ }^{\mathrm{TM}}$ device compared to other techniques, such as saline injection ${ }^{17}$ or pleural blood patching,[18] is that the BioSentry ${ }^{\mathrm{TM}}$ device is linked to standardized indications for use, deployment, and mechanisms of action. The technique of injection of saline or autologous blood into the biopsy tract is operator dependent and thus subject to variations in practice which in turn may account for why some clinical studies using the same techniques return conflicting results. In contrast, there is a standard technique used to deploy the BioSentry ${ }^{\mathrm{TM}}$ device, a dessicated polyethylene glycol hydrogel that deploys as a solid cylinder $(2.5 \mathrm{~cm}$ in length by $0.1 \mathrm{~cm}$ in diameter) and selfexpands upon contact with moist tissue to fill the void created by the biopsy needle.[19] The standard elements associated with the BioSentry ${ }^{\mathrm{TM}}$ device may account for why the three studies examining its use have been able to demonstrate either a reduction in pneumothorax rate, chest tube insertion rate or both.[19, 22], present study

Image-guided percutaneous biopsies have increased over time with radiologists being the physician specialty group providing the most biopsy services.[23] Using Medicare claims data from 1997-2008, the total number of biopsies performed by radiologists increased at a compound annual growth rate of $8 \%$, and radiologists' share of all biopsies increased from $35 \%$ to $56 \%{ }^{23}$ The use of low dose CT for lung cancer screening ${ }^{24}$, rise in availability of advanced imaging techniques for procedural guidance ${ }^{23}$, and the proliferation of targeted therapies in personalized cancer medicine ${ }^{25}$ are but a few factors contributing to this trend of increasing biopsy volume. Indeed, advances in CT technique have evolved so that small nodules which may represent early lung cancer are routinely detected and as a corollary, the professional society guidelines for biopsy are to consider tissue sampling for nodules that are $\geq 8 \mathrm{~mm} .{ }^{26,27}$ Moreover, clinical trials for emerging targeted therapies now often require tissue sampling for therapy stratification or at the very least, development of a companion diagnostic test. ${ }^{28,29}$ As radiologists are the largest physician provider group to perform image-guided biopsies, it becomes our responsibility to evaluate and consider ways of improving patient safety and decreasing complication rates.

While pneumothorax is the most common complication resulting from lung biopsy, a review of 660 lung biopsies demonstrated that a radiologist's experience level with lung biopsies is a major risk factor for pneumothorax occurrence: the more experience operator had a significantly lower pneumothorax rate of $17 \%$ as compared to the other radiologists (average pneumothorax rate, $30 \%) .{ }^{6}$ The BioSentry ${ }^{\mathrm{TM}}$ device may represent a standardized technique to overcome the learning curve associated with CT-guided lung biopsies and could potentially be used as a quality improvement tool to both narrow the performance band and also to shift the complications curve to result in lower rates of pneumothorax-related 
complications. Future studies could also focus on evaluating the potential costs benefits that could be associated with reducing the occurrence of pneumothorax, chest tube insertion rates and associated hospital stays.

Our study is not without limitations. Despite the propensity score matching analysis, this remains a retrospective study involving interventional radiologists ranging in spectrum in terms of their levels of experience with lung biopsies. The decision of when to place a BioSentry ${ }^{\mathrm{TM}}$ device was based on operator preference as no strict inclusion or exclusion criteria were mandated. In addition, we included historical cases in the control group which were done before the time BioSentry ${ }^{\mathrm{TM}}$ device became available for use; however, this was done because we needed to find a larger patient population in the control group to enable us to find sufficient propensity score matched patients in both treatment groups. This study did not evaluate if there was a learning curve associated with using the BioSentry ${ }^{\mathrm{TM}}$ device nor whether use of the device affected overall procedure time. Despite these limitations, the study clearly shows that the use of the self-expanding tract sealant device significantly reduced the pneumothorax rate, and more importantly, the chest tube placement rate after percutaneous lung biopsy.

\section{References}

1. Gurley MB, Richli WR, Waugh KA. Outpatient management of pneumothorax after fine needle aspiration: economic advantages for the hospital and patient. Radiology. 2998; 209(3):717-722.

2. Malone LJ, Stanfill RM, Wang H, Fahey KM, Bertino RE. Effect on intraparenchymal blood patch on rates of pneumothorax and pneumothorax requiring chest tube placement after percutaneous lung biopsy. AJR Am J Roentgenol. 2013; 200(6):1238-1243. [PubMed: 23701059]

3. Wiener RS, Schwartz LM, Woloshin S, Welch HG. Population-based risk of complications after transthoracic needle lung biopsy of a pulmonary nodule: an analysis of discharge records. Ann Intern Med. 2011; 155(3):137-144. [PubMed: 21810706]

4. Covey AM, Gandhi R, Brody LA, Getrajdman G, Thaler HT, Brown KT. Factors associated with pneumothorax and pneumothorax requiring treatment after percutaneous lung biopsy in 443 consecutive patients. J Vasc Interv Radiol. 2004; 15(5):479-483. [PubMed: 15126658]

5. Hiraki T, Mimura H, Gobara H, et al. Incidence of and risk factors for pneumothorax and chest tube placement after CT fluoroscopy-guided percutaneous lung biopsy: retrospective analysis of the procedures conducted over a 9-year period. AJR Am J Roentgenol. 2010; 194(3):809-814. [PubMed: 20173164]

6. Yeow KM, Su IH, Pan KT, et al. Risk Factors of pneumothorax and bleeding: multivariate analysis of 660 CT-guided coaxial cutting needle lung biopsies. Chest. 2004; 126(3):748-754. [PubMed: 15364752]

7. Geraghty PR, Kee ST, McFarlane G, et al. CT-guided transthoracic needle aspiration biopsy of pulmonary nodules: needle size and pneumothorax rate. Radiology. 2003; 229(2):475-481. [PubMed: 14595149]

8. Kim JI, Park CM, Lee SM, Goo JM. Rapid needle-out patient-rollover approach after cone beam CT-guided lung biopsy: effect on pneumothorax rate in 1,191 consecutive patients. Eur Radiol. 2015; 25(7):1845-1853. [PubMed: 25636421]

9. McCartney R, Tait D, Stilson M, Seidel GF. A technique for the prevention of pneumothorax in pulmonary aspiration biopsy. Am J Roentgenol Radium Ther Nucl Med. 1974; 120(4):872-875.

10. McCartney RL. Further observations on the lung patch technique: with analysis of the first 50 cases. Am J Roentgenol Radium Ther Nucl Med. 1975; 124(3):397-403.

11. Bourgouin PM, Shepard JA, McLoud TC, Spizarny DL, Dedrick CG. Transthoracic needle aspiration biopsy: evaluation of the blood patch technique. Radiology. 1988; 166(1 Pt 1):93-95. [PubMed: 3336708] 
12. Herman SJ, Weisbrod GL. Usefulness of the blood patch technique after transthoracic needle aspiration biopsy. Radiology. 1990; 176(2):395-397. [PubMed: 2367653]

13. Engeler CE, Hunter DW, Castaneda-Zuniga W, Tashjian JH, Yedlicka JW, Amplatz K. Pneumothorax after lung biopsy: prevention with transpleural placement of compressed collagen foam plugs. Radiology. 1992; 184(3):787-789. [PubMed: 1509068]

14. Moore EH, Shelton DK, Wisner ER, Richardson ML, Bishop DM, Brock JM. Needle aspiration lung biopsy: reevaluation of the blood patch technqiue in an equine model. Radiology. 1995; 196(1):183-186. [PubMed: 7784564]

15. Petsas T, Siamblis D, Giannakenas C, et al. Fibrin glue for sealing the needle tract in fine-needle percutaneous lung biopsy using a coaxial system. Part II. Clinical Study. Cardiovasc Intervent Radiol. 1995; 18(6):378-382. [PubMed: 8591624]

16. Lang EK, Ghavami R, Schreiner VC, Archibald S, Ramirez J. Autologous blood clot seal to prevent pneumothorax at CT-guided lung biopsy. Radiology. 2000; 216(1):93-96. [PubMed: 10887232]

17. Billich C, Muche R, Brenner G, et al. CT-guided lung biopsy: incidence of pneumothorax after instillation of NaCL into the biopsy tract. Eur Radiol. 2008; 18(6):1146-1152. [PubMed: 18270713]

18. Wagner JM, Hinshaw JL, Lubner MG, et al. CT-guided lung biopsies: pleural blood patching reducees the rate of chest tube placement for postbiopsy pneumothorax. AJR Am J Roentgenol. 2011; 197(4):783-788. [PubMed: 21940564]

19. Zaetta JM, Licht MO, Fisher JS, Avelar RL. A lung biospy tract plug for reduction of postbiopsy pneumothorax and other complications: results of a propsective, multicenter, randomized, controlled clinical study. J Vasc Interv Radiol. 2010; 21(8):1235-1243. [PubMed: 20656223]

20. Gupta S, Hicks ME, Wallace MJ, Ahrar K, Madoff DC, Murthy R. Outpatient management of postbiopsy pneumothorax with small-caliber chest tubes: factors affecting the need for prolonged drainage and additional interventions. Cardiovasc Intervent Radiol. 2008; 31(2):342-348. [PubMed: 18074173]

21. Kuban JD, Tam AL, Huang SY, et al. The effect of needle gauge on the risk of pneumothorax and chest tube placement after percutaneous computed tomographic (CT)-guided lung biopsy. Cardiovasc Intervent Radiol. 2015 Apr 30. [Epub ahead of print].

22. Grage RA, Keogh S, Naveed MA. Abstract No. 331-Comparison analysis pre and post implementation of a BioSentry tract sealant system after percutaneous transthoracic CT guided needle biopsy. J Vasc Interv Radiol. 2015; 26(2):S149.

23. Kwan SW, Bhargavan M, Kerlan RK Jr, Sunshine JH. Effect of advanced imaging technology on how biopsies are done and who does them. Radiology. 2010; 256(3):751-758. [PubMed: 20587643]

24. Wiener RS, Wiener DC, Gould MK. Risks of transthoracic needle biopsy: how high? Clin Pulm Med. 2013; 20(1):29-35. [PubMed: 23525679]

25. Kim ES, Herbst RS, Wistuba II, et al. The BATTLE trial: personalizing therapy for lung cancer. Cancer Discov. 2011; 1(1):44-53. [PubMed: 22586319]

26. Gould MK, Fletcher J, Iannettoni MD, et al. Evaluation of patients with pulmonary nodules: when is it lung cancer?: AACP evidence-based clinical practice guidelines ( $2^{\text {nd }}$ edition). Chest. 2007; 132(3 Suppl):108S-130S. [PubMed: 17873164]

27. MacMahon H, Austin JH, Gamsu G, et al. Guidelines for management of small pulmonary nodules detected on CT scans: a statement from the Fleischner Society. Radiology. 2005; 237(2):395-400. [PubMed: 16244247]

28. Overman MJ, Modak J, Kopetz S, et al. Use of research biopsies in clinical trials: are risk and benefits adequately discussed? J Clin Oncol. 2013; 13(1):17-22.

29. Tam AL, Kim ES, Lee JJ, et al. Feasiblity of image-guided transthoracic core-needle biopsy in the BATTLE lung trial. J Thorac Oncol. 2013; 8(4):436-442. [PubMed: 23442309] 


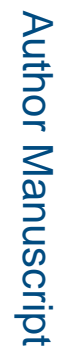

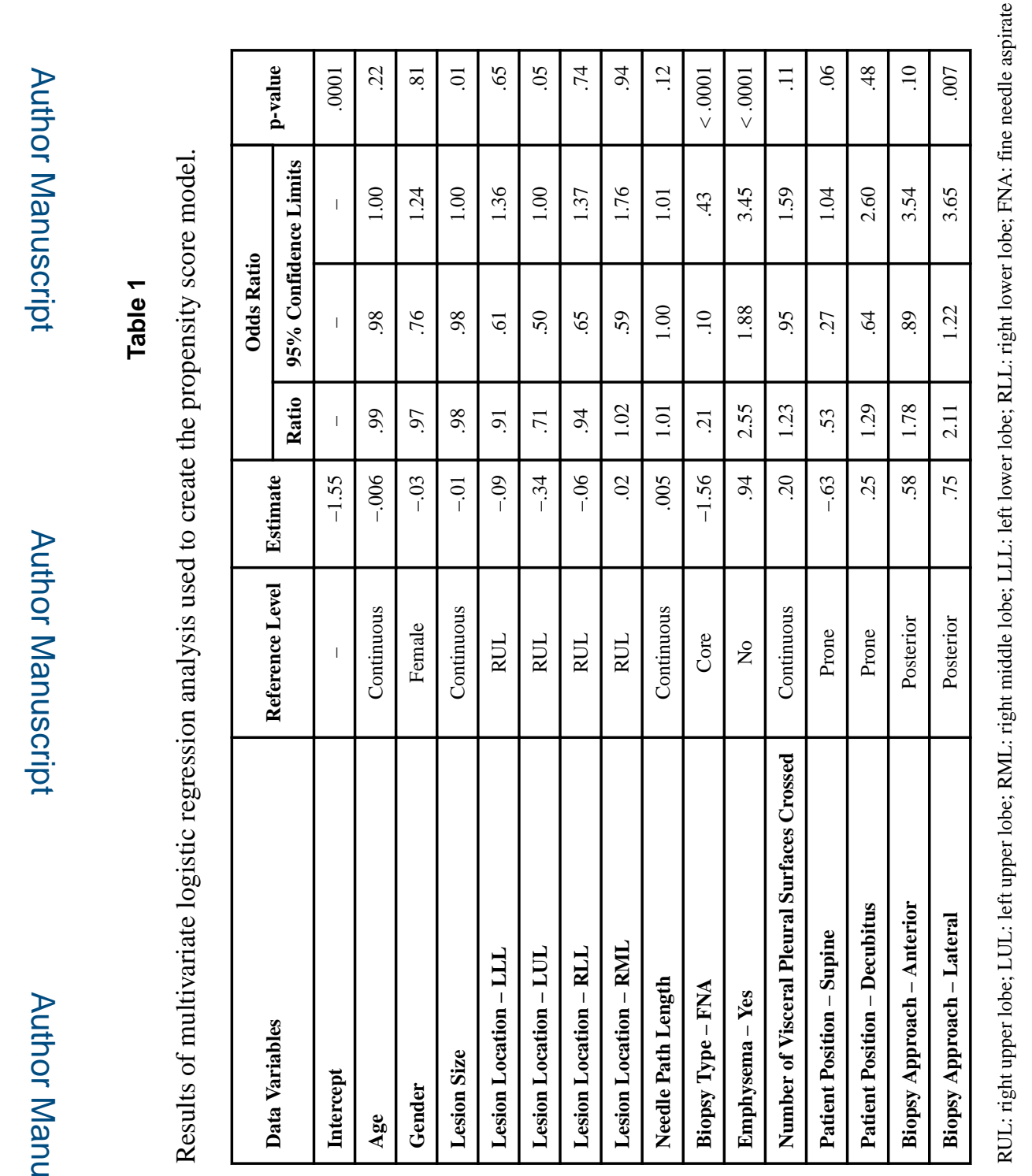

Cardiovasc Intervent Radiol. Author manuscript; available in PMC 2018 February 01. 


\section{Table 2}

Observed baseline patient-, lesion-, and procedure-specific characteristics stratefied by unmatched and matched data.

\begin{tabular}{|c|c|c|c|c|}
\hline \multirow{2}{*}{ Data Variable } & \multicolumn{2}{|c|}{ Unmatched Analysis } & \multicolumn{2}{|c|}{ Matched Analysis } \\
\hline & $\begin{array}{l}\text { Biosentry }^{\mathrm{TM}} \\
(\mathbf{n}=\mathbf{3 1 8})\end{array}$ & $\begin{array}{c}\text { Control } \\
(n=1956)\end{array}$ & $\begin{array}{c}\text { Biosentry }^{\mathrm{TM}} \\
(\mathbf{n}=\mathbf{3 1 7})\end{array}$ & $\begin{array}{l}\text { Control } \\
(\mathbf{n}=317)\end{array}$ \\
\hline Age (years) & 63.3 & 63.3 & 63.3 & 63.4 \\
\hline *Sex - Male & $49.1 \%$ & $49.6 \%$ & $49.1 \%$ & $52.1 \%$ \\
\hline Lesion Size (mm) & 19.8 & 23.0 & 19.8 & 21.2 \\
\hline${ }^{*}$ Lesion Location - LLL & $18.2 \%$ & $19.2 \%$ & $18.2 \%$ & $18.0 \%$ \\
\hline "Lesion Location - LUL & $20.1 \%$ & $26.1 \%$ & $20.1 \%$ & $25.2 \%$ \\
\hline *Lesion Location - RLL & $23.9 \%$ & $21.7 \%$ & $23.9 \%$ & $16.7 \%$ \\
\hline * Lesion Location - RML & $6.3 \%$ & $6.2 \%$ & $6.3 \%$ & $6.3 \%$ \\
\hline Needle Path Length (mm) & 31.3 & 27.9 & 31.3 & 33.2 \\
\hline *Biopsy Type -FNA & $2.5 \%$ & $10.1 \%$ & $2.5 \%$ & $2.2 \%$ \\
\hline *Emphysema - Yes & $26.1 \%$ & $11.9 \%$ & $26.1 \%$ & $27.4 \%$ \\
\hline Number of Visceral Pleural Surfaces Crossed & 1.15 & 1.10 & 1.15 & 1.18 \\
\hline *Patient Position - Supine & $41.2 \%$ & $44.3 \%$ & $41.2 \%$ & $44.2 \%$ \\
\hline *Patient Position - Decubitus & $4.1 \%$ & $2.4 \%$ & $4.1 \%$ & $3.2 \%$ \\
\hline *Biopsy Approach - Anterior & $30.2 \%$ & $33.7 \%$ & $30.2 \%$ & $32.2 \%$ \\
\hline *Biopsy Approach - Lateral & $18.2 \%$ & $14.0 \%$ & $18.2 \%$ & $18.3 \%$ \\
\hline
\end{tabular}

Denotes variables where the data is reported as a percentage.

RUL: right upper lobe; LUL: left upper lobe; RML: right middle lobe; LLL: left lower lobe; RLL: right lower lobe; FNA: fine needle aspirate 
Table 3

Pneumothorax and chest tube rates in propensity matched patients for all faculty

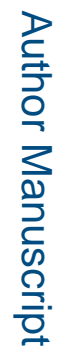

\begin{tabular}{|c|c|c|c|c|}
\hline & & \multicolumn{3}{|c|}{ No Biosentry } \\
\hline & & No PTx & PTx & Total \\
\hline \multirow{8}{*}{ Biosentry } & No PTx & $\begin{array}{r}167 \\
(52.68 \%)\end{array}$ & $\begin{array}{r}84 \\
(26.50 \%)\end{array}$ & $\begin{array}{r}251 \\
(79.18 \%)\end{array}$ \\
\hline & PTx & $\begin{array}{r}46 \\
(14.51 \%)\end{array}$ & $\begin{array}{r}20 \\
(6.31 \%)\end{array}$ & $\begin{array}{r}66 \\
(20.82 \%)\end{array}$ \\
\hline & Total & $\begin{array}{r}213 \\
(67.19 \%)\end{array}$ & $\begin{array}{r}104 \\
(32.81 \%)\end{array}$ & $\begin{array}{r}317 \\
(100.00 \%)\end{array}$ \\
\hline & & \multicolumn{3}{|c|}{ No Biosentry } \\
\hline & & No Tube & Tube & Total \\
\hline & No Tube & $\begin{array}{r}233 \\
(73.5 \%)\end{array}$ & $\begin{array}{r}58 \\
(18.30 \%)\end{array}$ & $\begin{array}{r}291 \\
(91.80 \%)\end{array}$ \\
\hline & Tube & $\begin{array}{r}18 \\
(5.68 \%)\end{array}$ & $\begin{array}{r}8 \\
(2.52 \%)\end{array}$ & $\begin{array}{r}26 \\
(8.20 \%)\end{array}$ \\
\hline & Total & $\begin{array}{r}251 \\
(79.18 \%)\end{array}$ & $\begin{array}{r}66 \\
(20.82 \%)\end{array}$ & $\begin{array}{r}317 \\
(100.00 \%)\end{array}$ \\
\hline
\end{tabular}

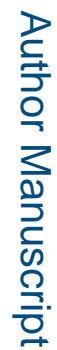

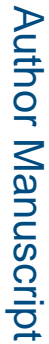




\section{Table 4}

Observed baseline patient-, lesion-, and procedure-specific characteristics stratefied by unmatched and matched data for the five faculty used for the sub-analysis.

\begin{tabular}{|c|c|c|c|c|}
\hline \multirow{2}{*}{ Data Variable } & \multicolumn{2}{|c|}{ Unmatched Analysis } & \multicolumn{2}{|c|}{ Matched Analysis } \\
\hline & $\begin{array}{c}\text { Biosentry }^{\mathrm{TM}} \\
(\mathbf{n}=\mathbf{2 3 0})\end{array}$ & $\begin{array}{l}\text { Control } \\
(\mathbf{n}=\mathbf{8 8 7})\end{array}$ & $\begin{array}{c}\text { Biosentry }^{\mathrm{TM}} \\
(\mathbf{n}=\mathbf{2 2 2})\end{array}$ & $\begin{array}{l}\text { Control } \\
(\mathrm{n}=222)\end{array}$ \\
\hline Age (years) & 63.5 & 63.3 & 63.5 & 63.1 \\
\hline *Sex - Male & $46.5 \%$ & $48.9 \%$ & $45.9 \%$ & $45.9 \%$ \\
\hline Lesion Size (mm) & 19.8 & 22.9 & 19.8 & 20.2 \\
\hline${ }^{*}$ Lesion Location - LLL & $19.1 \%$ & $19.4 \%$ & $19.4 \%$ & $16.8 \%$ \\
\hline${ }^{*}$ Lesion Location - LUL & $18.7 \%$ & $25.9 \%$ & $18.9 \%$ & $22.5 \%$ \\
\hline *Lesion Location - RLL & $24.4 \%$ & $21.8 \%$ & $24.3 \%$ & $24.3 \%$ \\
\hline * Lesion Location - RML & $6.1 \%$ & $6.7 \%$ & $6.3 \%$ & $6.8 \%$ \\
\hline Needle Path Length (mm) & 30.2 & 26.7 & 29.9 & 31.9 \\
\hline *Biopsy Type -FNA & $2.6 \%$ & $9.5 \%$ & $2.7 \%$ & $3.2 \%$ \\
\hline${ }^{*}$ Emphysema - Yes & $26.1 \%$ & $11.8 \%$ & $24.3 \%$ & $23.4 \%$ \\
\hline Number of Visceral Pleural Surfaces Crossed & 1.1 & 1.1 & 1.1 & 1.1 \\
\hline 'Patient Position - Supine & $40.9 \%$ & $44.5 \%$ & $41.0 \%$ & $42.8 \%$ \\
\hline *Patient Position - Decubitus & $3.9 \%$ & $2.1 \%$ & $3.2 \%$ & $2.3 \%$ \\
\hline *Biopsy Approach - Anterior & $30.0 \%$ & $32.5 \%$ & $29.7 \%$ & $32.4 \%$ \\
\hline *Biopsy Approach - Lateral & $19.1 \%$ & $15.5 \%$ & $18.5 \%$ & $18.5 \%$ \\
\hline
\end{tabular}

Denotes variables where the data is reported as a percentage.

RUL: right upper lobe; LUL: left upper lobe; RML: right middle lobe; LLL: left lower lobe; RLL: right lower lobe; FNA: fine needle aspirate 


\section{Table 5}

Pneumothorax and chest tube rates in propensity matched patients for the five faculty used for the sub-analysis

\begin{tabular}{|l|l|r|r|r|}
\hline \multicolumn{2}{|c|}{} & \multicolumn{3}{|c|}{ No Biosentry } \\
\cline { 2 - 5 } & No PTx & PTx & Total \\
\hline \multirow{4}{*}{ No PTx } & 130 & 53 & 183 \\
& $(58.56 \%)$ & $(23.87 \%)$ & $(82.43 \%)$ \\
\cline { 2 - 5 } & PTx & 25 & 14 & 39 \\
& $(11.26 \%)$ & $(6.31 \%)$ & $(17.57 \%)$ \\
\cline { 2 - 5 } & Total & 155 & 67 & 222 \\
& & $(69.82 \%)$ & $(30.18 \%)$ & $(100.00 \%)$ \\
\cline { 2 - 5 } & & \multicolumn{3}{|c|}{ No Biosentry } \\
\cline { 2 - 5 } & & No Tube & Tube & Total \\
\cline { 2 - 5 } & No Tube & 170 & 36 & 206 \\
& & $(76.58 \%)$ & $(16.22 \%)$ & $(92.79 \%)$ \\
\cline { 2 - 5 } & Tube & 12 & 4 & 16 \\
& $(5.41 \%)$ & $(1.80 \%)$ & $(7.21 \%)$ \\
\cline { 2 - 5 } & Total & 182 & 40 & 222 \\
& $(81.98 \%)$ & $(18.02 \%)$ & $(100.00 \%)$ \\
\hline
\end{tabular}

